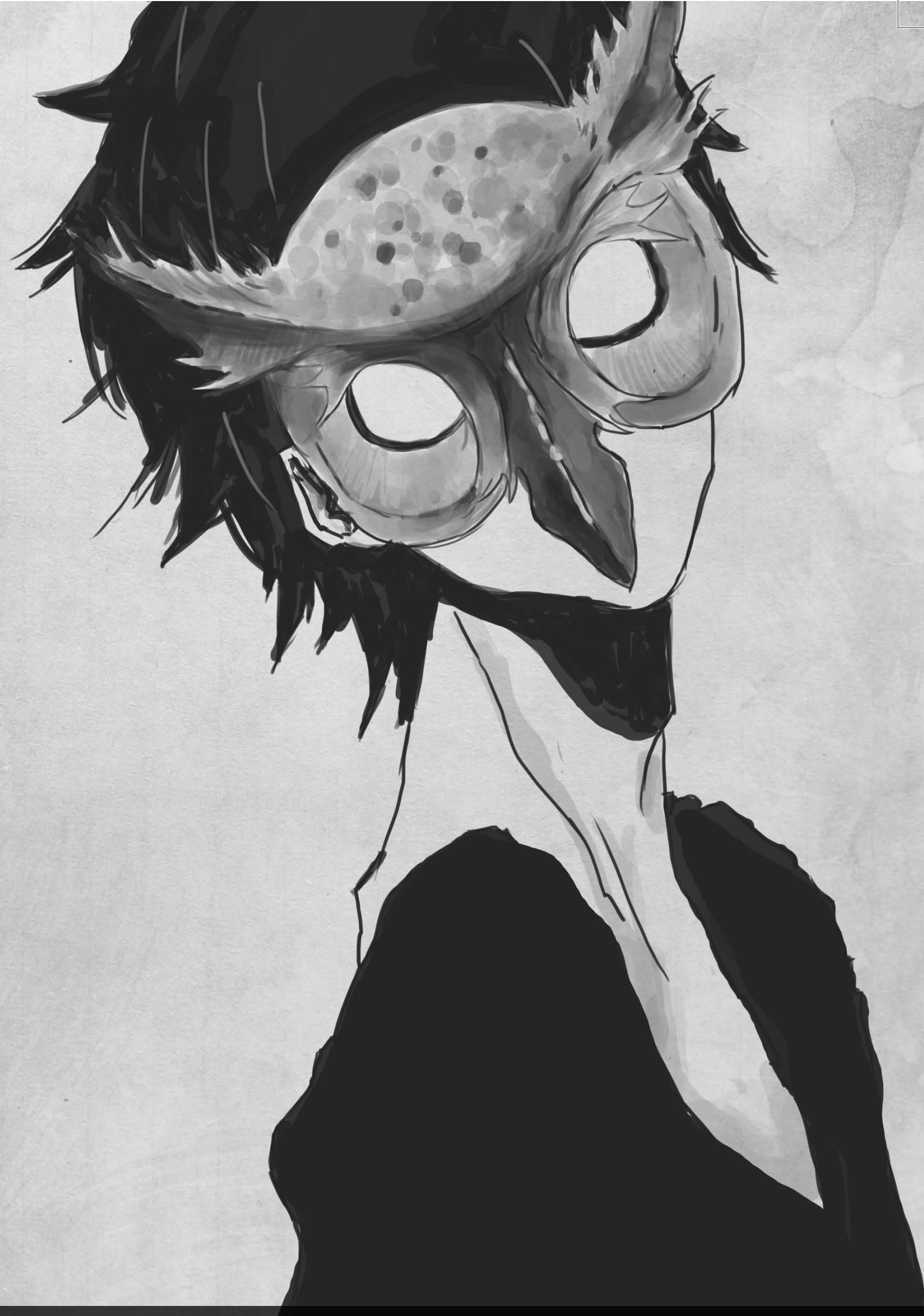

Condiciones de posibilidad para una mirada a la subjetividad del maestro en Colombia 


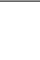




\section{Condiciones de posibilidad para una mirada a la subjetividad del maestro en Colombia}

Fecha de recepción: 28 de julio de 2011

\section{Luis Ernesto Vásquez Alape}

Universidad de La Salle

Ivasquez@unisalle.edu.co

Docente investigador de la Facultad de Educación

de la Universidad de La Salle. Integrante del grupo de Investigación de Colciencias

"Pedagogía,cultura y formación docente" y del

Centro de investigación en educación y pedagogía, CIEP.

\section{Resumen}

La preocupación por rescatar la reflexión sobre la subjetividad del maestro, desde una mirada cimentada en la filosofía política a partir de un enfoque arqueogenealógico, es el principal eje dinamizador de este escrito, para ello se hace una disertación generada en la investigación sobre la salud mental del maestro en Colombia que se ha iniciado en la Facultad de Educación de la Universidad de La Salle. Este escrito intenta mostrar emergencias y procedencias $^{1}$ (Foucault, 2002) y condiciones de posibilidad históricas en las que se produce la subjetividad del maestro en Colombia desde la implementación de racionalidades, tecnologías y dispositivos de poder que afectan sus prácticas y la calidad educativa.

\section{Palabras clave}

Condición de posibilidad, subjetividad del maestro, ontología crítica.

$1 \quad$ Emergencia y procedencia son dos nociones que acuña Foucault al construir su método de análisis de la historia a partir de la lectura que hace sobre Nietzsche. El primero permite mirar la multiplicidad de huellas que entrecruzadas conforman la subjetividad del maestro, mostrando su procedencia heterogénea, por ser experiencia histórica. La segunda supone "la entrada en escena de las fuerzas" de donde emerge el suceso 0 , de manera más concreta, el sujeto maestro como suceso.

The concern to rescue the reflection on the subjectivity of the teacher from a perspective grounded in political philosophy from a focus arqueogenealógico dynamic is the main hub of this writing, for it is a dissertation research generated in the teacher's mental health in Colombia, which started in the Faculty of Education at the University of La Salle. This paper attempts to show the arising, origin (Foucault, 2002) and historical conditions of possibility which producs the subjectivity of the teacher in Colombia since the implementation of rationalities, technologies and systems of power that affect their practices and educational quality.

\section{Keywords}

Condition of possibility, subjectivity of the teacher, critical ontology. 


\section{Intención}

La intención de este trabajo es proponer una reflexión crítica sobre la subjetividad del maestro colombiano a partir de un análisis cimentado en la filosofia política legitimada por Foucault. Este análisis se hará desde su arqueogenealogía como proceso escrutador de los diferentes estratos, capas y sedimentos que constituyen la subjetividad del maestro como fruto de los múltiples entrecruzamientos económicos, políticos, sociales y culturales evidenciados en sus prácticas históricas y/o historizantes.

Dentro de las prácticas politicas, educativas y modos de subjetivación contemporáneos en Colombia, a los maestros se nos constituye en sujetos de calidad educativa a partir de capacitaciones y/o procesos formativos en modelos pedagógicos, currículo, planeación, proyectos educativos, lectura y escritura didácticas, evaluación, psicología del niño, psicología del joven y politicas públicas, entre otros, con el fin de mejorar las prácticas didácticas y responder a unas competencias de productividad e indicadores de calidad. Pero realmente son escasos, por no decir nulos, los espacios que se ofrecen o se construyen para que el maestro reflexione o se piense a sí mismo de manera categorialmente organizada, como sujeto histórico, ético/ estético, político y sujeto educativo que tiene la posibilidad de constituirse desde una "ontologia crítica del presente" a partir de la práctica de una "ontología crítica del sí mismo"(Foucault, 1985). Este es un vacío fundamental si se considera que el maestro es, no sólo la principal mediación pedagógica, sino un ser humano, sujeto histórico y hacedor de historia que desde su existencia, su quehacer y saber pedagógicos tiene incidencia en la calidad educativa, entendida no como dispositivo ${ }^{2}$ o estrategia para la generación de sujetos instrumentalizados en pro de la productividad económica, sino como sujetos de calidez de vida, en el sentido

2 De acuerdo con las reflexiones que comienza a hacer Foucault hacia 1977 sobre lo que él llamó la gubernamentalidad o el gobierno de los hombres, se puede comprender el dispositivo como 1) Un conjunto heterogéneo que incluye virtualmente cualquier cosa, lo lingüístico y lo no-lingüístico, al mismo título: discursos, instituciones, edificios, leyes, medidas de policía, proposiciones filosóficas, etc. El dispositivo en sí mismo es la red que se establece entre estos elementos. 2) El dispositivo tiene siempre una función estratégica concreta y se inscribe siempre en una relación de poder. 3) Es algo general, un reseau, una "red", porque incluye en sí la episteme, que es, para Foucault, aquello que en determinada sociedad permite distinguir lo que es aceptado como un enunciado científico de lo que no es científico (Agamben). de comprenderse como sujetos de desujeción, sujetos de libertad.

El sujeto-maestro, como sujeto de libertad, a partir de su quehacer y saber, se constituye en la principal mediación pedagógica que ha de favorecer la consolidación de pensamiento crítico, creativo, reflexivo y, por ende, investigativo, en sus estudiantes, a partir de la configuración de ambientes -medios- pedagógicos favorecedores de prácticas y órdenes de discursos que, antes que favorecer la repetición y domesticación de ideas, afectos y desafectos, debe facilitar la constitución de ambientes pedagógicos en donde se dé vía libre al discurso, al discurrir de cuestionamientos en el sentido de la cualificación del pensamiento, "de la sospecha", al tiempo que a la configuración de una ética como estética de la existencia.

Este artículo surge de las primeras reflexiones suscitadas por la investigación "La salud mental del sujeto maestro en Colombia", que se circunscribe al campo de subjetividad y verdad (Foucault, 2002) y se ubica en la linea de investigación "Filosofia, praxis y calidad educativa" de la Facultad de Educación de la Universidad de La Salle. Se trata de refle-xionar, a partir de una mirada tanto ética como estética de la existencia, en torno a las relaciones de saber-poder-verdad que constituyen al sujeto maestro como un sujeto vulnerable desde el punto de vista de la salud mental.

Este escrito se limitará a versar sobre algunos aspectos que, desde prácticas históricas y racionalidades, se han convertido en condición de posibilidad en algunos de los modos de subjetivación por los que ha pasado el maestro en Colombia, mas no ahondará en el problema del maestro como sujeto de salud mental.

Se trata entonces, de mostrar, en un primer momento, condiciones de posibilidad que entrecruzan al sujeto constituyéndolo históricamente como sujeto de mendicidad (Martínez, 2002) de instrucción pública (Matínez,1984); sujeto, agente social (Saldarriaga, 2003) y sujeto de progreso, para ubicar, más adelante, la manera como estas dinámicas de sujeción con sus tecnologías, dispositivos y estrategias inciden en el sujeto maestro como sujeto de salud mental y/o sujeto de enfermedad.

En esta reflexión se presentará una retrospectiva sobre algunos modos de subjetivación del 
maestro en nuestra historia colombiana; para ello se tomarán momentos históricos siguiendo la lógica analítica de las discontinuidades ${ }^{3}$. En esta reflexión se toman dos casos: el de la procedencia y emergencia del maestro como sujeto público en la Colonia y el de la constitución del mismo a finales del siglo XIX y comienzos del $\mathrm{XX}$, en medio del entrecruzamiento de racionalidades soberanas, pastorales y estatales. La pregunta que surge aquí es ¿Cómo estas diversas prácticas y racionalidades constituyen la subjetividad del maestro?

\section{Acercamiento a la procedencia y emergencia del maestro en Colombia}

La emergencia del maestro público en Colombia, que se comienza a gestar hacia la segunda mitad del siglo XVIII en la Nueva Granada, se produce en medio del cambio de racionalidad que agencian los Borbones dada la competencia por la hegemonía comercial entre España, Holanda, Francia e Inglaterra. Esto obliga a que se muten las prácticas y tecnologías políticas de gobierno, dando paso a lo que Foucault llama "la razón de Estado". Ésta se caracteriza por concentrar todas las prácticas sociales, potenciar la vida, garantizar la utilización óptima de los recursos, y ejercer una adecuada administración de la población con el fin de "crear las condiciones para (...) ejercitar una politica de control sobre las instituciones sociales, sobre los recursos naturales y, por encima de todo, sobre la vida de los súbditos" (Castro, 2007, p. 99), de tal forma que garanticen la centralización del poder y el crecimiento económico.

Se produce, de esta manera, la instauración de "un arte de gobernar autónomo y específico, diferente del ejercicio de la soberanía y también de la gestión pastoral (...) ese arte de gobernar de presunta existencia, es de un tipo diferente a las leyes de Dios y a las leyes de la naturaleza" (Foucault, 2006, p. 285). Factor por el cual, el nuevo Gobierno, restringe el poder de la Iglesia, el pensamiento especulativo y da preeminencia a la racionalidad de Estado, entendida como la racionalización de la práctica gubernamental en el ejercicio de la soberanía politica, al tiempo que, en el campo del conocimiento,

3 La discontinuidad en términos de la arqueología de Foucault supone no seguir los datos históricos de manera lineal y continua, pues de lo que se trata es de mirar en las fisuras de la historia hechos que desde prácticas concretas constituyen al sujeto (lo sujetan), en el fluir de dispositivos de podersaber engranados desde racionalidades. fortalece las prácticas y discursos científicos quitando protagonismo a las prácticas discursivas doctrinarias de carácter religioso.

El poder y la seguridad ya no están garantizados por la tierra, sino por la adecuada administración sobre los hombres: "los borbones observan que la verdadera riqueza de las naciones no está primeramente (sic) en los recursos naturales sino en los recursos humanos disponibles" (Castro, 2007, p. 99).

Es en este contexto y en medio de los procesos de modernización del Estado, en que, para recuperar el poder, los Borbones expulsan a los jesuitas al tiempo que autorizan la creación de la escuela pública (a diferencia de la escuela Pia) y el nombramiento oficial de maestros, quienes deben enseñar a leer, escribir y contar y cumplir con requisitos como los de "ser hombre blanco y decente, arreglado, de buen procedimiento y sin vicio alguno" (Archivo Histórico Nacional, Tomo I, citado por Martinez, 1995).

\section{El devenir del maestro}

El devenir del maestro público y sus diferentes modos de constitución, que se dan en el entrecruzamiento de tecnologias politicas, dispositivos, estrategias y prácticas politicas, sociales, culturales y educativas atravesadas por relaciones de poder-saber, hacen parte fundamental de las "genealogias de la colombianidad" fluido de procedencias y emergencias entretejidas en relaciones de saberpoder pertenecientes a regimenes de verdad y a tendencias y lineas de fuerza que han conducido hacia la preeminencia del tipo de poder que podemos llamar gobierno sobre todos los demás: soberania, disciplina y que inducen, por un lado el desarrollo de toda una serie de aparatos específicos de gobierno, y por otro, el desarrollo de una serie de saberes (Foucault, 2006, p. 136) que han constituido la subjetividad del maestro

$4 \quad$ Neologismo acuñado por Santiago Castro, desde donde propone "lo colombiano", no como algo estático sino que se va constituyendo día a día en el entrecruzamiento de sus múltiples prácticas. 
como sujeto de productividad dirigida y de autonomía perdida $5^{5^{*}}$

A lo largo de la historia en Colombia, el maestro ha sido producido por diferentes regímenes discursivos, tendencias, líneas de fuerza, prácticas y tecnologias gubernamentales de acuerdo con una serie (y serie de series) de condiciones de posibilidad históricas que lo atraviesan. Dentro de las investigaciones realizadas por el grupo de historia de la práctica pedagógica en Colombia, aparece como sujeto de saber pedagógico constituido por matrices, saberes, estrategias y dispositivos (Saldarriaga, 2003) que lo sujetan y controlan y/o, de acuerdo con las circunstancias históricas, lo han acercado a prácticas de desujeción como en el caso de la emergencia del Movimiento Pedagógico en la década de los ochenta.

El maestro aparece como "sujeto del desa-rraigo", en la Colonia (Martínez, Boom et al., 1995) y como "salvador de la patria" o agenciador de lo social entre fines del siglo XIX y comienzos del XX (Saldarriaga, 2003). De otra parte, también se le ha signado como transmisor de valores y conocimientos impuestos por la sociedad de consumo que busca reproducirse histórica e intergeneracionalmente, maestro apóstol, asalariado, maestro taylorizado, intelectual subordinado, lider social, trabajador de la cultura, entre otros: "un individuo cruzado por el desarraigo y desprovisto de poder, al que siempre los intelectuales, la Iglesia, el Estado, la familia, las instituciones le han exigido grandes responsabilidades, e impuesto tareas heroicas" (Martínez, 1984, p. 246). Hacia la década del ochenta, con el surgimiento del Movimiento Pedagógico, se le enuncia como sujeto político, sujeto de saber pedagógico y trabajador de la cultura.

Los "modos de ver" y "las maneras de decir" actuales constituyen al maestro como sujeto de funciones, producto de la tecnologia neolibe-ral: las prácticas discursivas que lo producen tienen que ver con tecnologías políticas, juegos de verdad y relaciones de poder que buscan ajustar de la manera más perfecta posible el ejercicio global del poder político a los principios de una economía de mercado, para construir "un Estado bajo vigilancia del mercado más que un mercado bajo la vigilancia del Estado" (Foucault, 2008,

$5 \quad$ * Comentario a los contenidos del texto "Crónicas del desarraigo de Martínez Boom, Jorge Orlando Castro y Carlos Noguera", escrito por Alberto Echeverry. p. 149). Este proceso exige la implantación de nuevas formas de gobierno de la vida, entre las que desempeñan un papel esencial la privatización de los mecanismos de seguridad social, la extensión del modelo de empresa -el sujeto como "empresario de sí mismo"- y la teoría del capital humano, que se evidencian en las prácticas del maestro. Estas lo han instrumentalizado a tal punto que el espacio para la reflexión crítica es limitado, trayendo como consecuencia la cosificación de sus acciones, de su subjetividad y la proyección de la misma en la comunidad educativa.

A partir de este ángulo de visión, la aparición del maestro en cada circunstancia histórica en Colombia se da por utilidad politica, en la medida en que, desde el punto de vista del Estado, éste no existe más que para aportar una transformación, aunque sea minima, a su potencia. El Estado no debe, pues, ocuparse del maestro más que en la medida en que él pueda introducir un cambio; a veces le pide vivir, trabajar, producir y consumir, y otras veces le pide morir. De esta manera, en la línea de Foucault (2004) el individuo se convierte en tecnologia politica de marginalidad politica, que responde a una racionalidad de Estado que sirve a efectos del poder de gobierno).

\section{Producción y emergencia del maestro}

Las prácticas sociales y culturales de una parte, y las tecnologías políticas de otra, atravesadas en diagonal por relaciones de saber-poder, constituyen al maestro en la historia colombiana como sujeto gubernamentalizado en la obediencia y la mediación para conducir los cuerpos y las poblaciones a través de la instrucción pública.

Desde el preciso momento en que comienza la conquista y prosigue la colonización de nuestro territorio y la territorialización de los cuerpos, esto es, el dominio y el sometimiento de las formas de ver y maneras de decir, aparece la figura de quien enseña, de quien forma, desde unos hábitos, costumbres y creencias occidentales que combinan la fuerza del guerrero con la comprensión y los buenos oficios del pastor. A partir de allí se imponen modelos 
de hegemonía/subalternidad que permiten agenciar $^{6}$ los cuerpos de los nativos, de tal manera que le respondan a la racionalidad del poder/saber dominante, aquel motivado por el proyecto de las cruzadas, con su deseo de expandir el cristianismo como religión universal (Todorov, 2007), al tiempo que encontrar fuentes de riqueza que permitan financiar su crisis económica.

Se inicia, de esta manera, el contacto y la interacción con otra cultura y otra visión de mundo a partir de los intereses, necesidades y conocimientos particulares de conquistadores y colonizadores que configuran al "otro" y constituyen un tipo de sujeto a partir de relaciones de poder-saber y verdad que pertenecen al "mundo civilizado".

Los conquistadores llegan y no se preguntan/ no preguntan por el nombre de los seres que alli existen, sino que los bautizan de acuerdo con la mirada constituida en su historia ("no existe otra"). ¿Qué es lo que motiva e impulsa a Colón para conquistar las nuevas tierras? En palabras de Todorov (2007), son tres móviles: el primero, la riqueza, el segundo, un móvil divino, y el tercero, el disfrute de la naturaleza (p.23). De otro lado, a Hernán Cortés le interesa de entrada la interpretación y comprensión del lenguaje de los indios para poder someterlos, manifestando, de esta manera, "una conciencia politica de sus actos" respaldada por su "servicio a Dios y al Rey" (p. 107). De otra parte está la visión del pastor, Fray Bartolomé de las Casas, quien demuestra que los indios tienen alma y que es necesario salvaguardarla desde los preceptos bíblicos.

En la época del descubrimiento del nuevo territorio por parte de Colón, el papado legitimaba las conquistas y se interesaba por darle sentido cristiano a los conquistados a partir del adoctrinamiento, al tiempo que los reyes católicos se comprometian con la conversión de los habitantes de las tierras subyugadas. "Las

$6 \quad$ Para Deleuze, todo agenciamiento es, en primer lugar, territorial. La primera regla concreta de los agenciamientos es descubrir la territorialidad que engloban pues siempre es una (...). El territorio está hecho de fragmentos decodificados de todo tipo, extraídos de los medios, pero que a partir de ese momento adquieren un valor de "propiedades": hasta los ritmos adquieren aquí un nuevo sentido (ritornelos). El territorio crea el agenciamiento. El territorio excede, a la vez, el organismo y el medio y la relación entre ambos; por ello el agenciamiento va también más allá del simple "comportamiento" (Deleuze). http://deleuzefilosofia.blogspot.com/2008/04/concepto-agenciamiento.html bulas Ínter caetera (1493) y Eximiae devotionis (1493 y 1501) de Alejandro VI, Universalis eclesiae (1508) de Julio II y Exponi novis (1523) de Adriano VI, otorgadas a la corona castellana, determinaron la estructura esencial del trabajo de evangelización en América" (Herrán, 1998, p. 9), de tal forma que las primeras "prácticas de enseñanza" que se efectuaron en la Nueva Granada, tuvieron como objeto el adoctrinamiento y no la instrucción, ni la enseñanza, ni la socialización, ni el progreso.

Como consecuencia de la expulsión de los jesuitas en el año de 1767, surgen en la Nueva Granada "sujetos que andan por las estancias pregonando enseñar a leer escribir y contar. Cambiaban su saber por un real, una vela y un pan semanal" (Martínez, Boom et al., 1995, p. 31). La educación formal que se impartía en los colegios quedó desprotegida, ante lo cual aquellas personas que habian recibido algún tipo de formación en primeras letras comenzaron a ofrecer sus servicios.

A partir del poder político y económico que habian logrado obtener los jesuitas tanto en España como en sus diferentes colonias, Carlos III, bajo la propuesta de modernización del Estado, emite el 2 de Abril de 1767 la "Pragmática sanción de su Magestad en fuerza de ley para el estrañamiento de estos Reynos a los Regulares de la Compañía, ocupación de sus Temporalidades, y prohibición de su restablecimiento en tiempo alguno, con las demás prevenciones que expresa" (Carlos III, Pragmática sanción de su majestad, 1767, tomado de: http://www.cervantes virtual. com). De esta manera, y bajo la égida de las Reformas Borbónicas, se propone una nueva concepción del Estado español sobre la base de la modernización de la Nación y la transformación de la sociedad en busca "del bienestar general". Esta ideología permitió el afianzamiento de una monarquía absoluta y el surgimiento del despotismo ilustrado, al tiempo que permitió la toma del control de la educación por parte del Estado, bajo la consigna de lo que le conviniera, ya que con esta acción se podía recuperar la gubernamentabilidad que hasta entonces tenian los jesuitas sobre las poblaciones, y los espacios de poder, vigilancia y control que se habian perdido.

Las determinaciones tomadas por la corona española crean crisis en el Nuevo Reino de Granada al cerrarse los colegios regentados por la Compañia de Jesús y quedar la 
población "huérfana" de profesores. Es en este contexto donde las circunstancias exigen la aparición del maestro público, la emergencia del maestro de escuela. Como primera manifestación de este tipo de sujeto enseñante, emerge el sujeto público mercader no autorizado para la enseñanza de las primera letras, aquel que ofrece la enseñanza pregonando sus servicios, constituyéndose, de esta manera, en el precursor del maestro público en la Nueva Granada. De manera simultánea a la aparición del sujeto "pregonero de la enseñanza", el Estado, en su proceso de recuperación del poder, propone una legislación donde declara que la enseñanza no podrá seguir perteneciendo a la familia y a la Iglesia como patrimonio autónomo e impenetrable y expresa taxativamente que "la enseñanza pública debe estar baxo la protección del príncipe" y que sólo a él como esencia del Estado es "a quien incumbe el cuidado y superintendencia de la educación de la juventud" (Colección General de Providencias, citado por Martínez, Boom et al., 1984, p. 34).

Se instaura la autorización virreinal, a través de la que se produce el nombramiento de maestros para la enseñanza de las prime-ras letras, este título se constituye en un mecanismo biopolítico que utiliza el poder estatal para vigilar, sujetar y controlar esta población de personas dedicadas a la enseñanza, al tiempo que a las poblaciones que se educan. Por medio del nombramiento de maestros, las autoridades virreinales ponen limite a aquella actividad que hasta entonces se ejercía libremente. La garantía sobre el saber del maestro no es lo que importa, lo que interesa, según la certificación expedida por el virreinato, son "la virtuosidad y buenas costumbres" Martinez, Castro, Noguera, 2002, p 46). Los requisitos exigidos al maestro implicaban una conducta religiosa regida por los principios de la moral cristiana. De esta manera, encontramos una superposición de poder pastoral, disciplinar y biopolitico mediante el que se dinamiza una anatomo y una biopolítica.

De otra parte, quienes presentan las solicitudes para ejercer como maestros, lo hacen para asegurar y garantizar su propio sustento. El período que va desde 1770 hasta 1800 está lleno de expedientes en los cuales se solicita el título de maestro.

Una vez se hacen los nombramientos oficiales de los maestros, comienza el problema con los pagos que se les deben dar por los servicios prestados pues se considera que es la comunidad que se beneficia y por lo tanto la que le ha de pagar al profesor. Sólo años más tarde, y gracias a la intervención de los vecinos en donde se encontraban ubicadas las escuelas, se autorizó pagar el sueldo del maestro con los fondos recaudados por el cabildo, proveniente de aquellos impuestos llamados "propios" que se impugnaban a las casas de juego y a las chicherias. Así lo expresa el Virrey Espeleta en su relación de mando de 1797 en la que haciendo alusión al estado de la instrucción pública informaba al Rey “(...) que en los lugares de afuera y de alguna población, se han establecido muchas [escuelas públicas] costeadas por las rentas de propios que en ésta tendrian una digna inversión" (Archivo Histórico Nacional de Madrid, citado por Martínez, 1995, p. 38).

Al dar un salto hasta fines del siglo XIX y comienzos del XX, Óscar Saldarriaga (2003) plantea que al maestro se le endosa la función de lo social. En el período comprendido entre 1870 y 1946 surge en medio de un campo de fuerzas y de tensiones en donde se entrecruzan permanentemente aspectos y categorias de diversas matrices constituyendo un tipo de sujeto social que se mueve entre la matriz de lo moral católico (pastoral), la de gestión de lo social desde la filosofia liberal, la biomédica en el siglo XIX, y la sociopolítica que hace su aparición en el siglo XX hacia 1914 (con la fundación del gimnasio Moderno) y se consolida con la Reforma Constitucional de 1936, en donde se favorece la Reforma Pedagógica, a la vez que se propugna por una "democratización de la cultura" y una "cultura aldeana" (Saldarriaga, 2003, p. 208).

Con la Reforma Radical de 1870 y la emisión del Decreto orgánico de instrucción pública primaria, se oficializa un proceso de gubernamentalización que legitima el gobierno sobre los individuos, la familia y la población, poniendo en diálogo el conocimiento, la ciencia, la formación en los valores patrios y el cuidado sobre los cuerpos, sin excluir la formación moral de carácter religioso. El Decreto orgánico funge como dispositivo de unidad a partir del que se gestiona la población y se garantiza la seguridad y la territorialización del Estado, avalando con esto "la constitución de redes por donde circula el poder: la primera es de orden microfísico en la que operarian tecnologias disciplinarias y de producción de sujetos, así como las 'tecnologías del yo' que buscan una producción autónoma de la subjetividad. Este sería el nivel de la corpopolitica. La segunda 
es de orden mesofísico en la cual se inscribe la gubernamentalidad del Estado y su control sobre las poblaciones. Este sería el nivel de la biopolitica" (Foucault, citado por Castro, 2005, p. 4). Se combina, de esta manera, racionalidad pastoral, soberana y de gubernamentalidad, para facilitar la injerencia del Estado en las vidas y en el poder de decisión de los individuos.

A partir de la razón de Estado, propuesta por la Reforma Radical y la gubernamentabilidad en la escuela, se crea la dirección general de instrucción pública que genera unas prácticas cimentadas en la enseñanza, la inspección y la administración. Dentro de la constante interacción generada por esta triada, se formulan los programas de enseñanza, se estructuran y se publican los textos, se organizan las bibliotecas escolares y se llevan a cabo las estadísticas, al tiempo que se "apropia" de la episteme que ha de guiar los saberes de los profesores a través del direccionamiento de las normales. Se trata de consolidar las normas y racionalizar los controles para caminar hacia la civilización, el progreso y la modernización de la Nación: "De aquí se derivan una serie de consecuencias; la soberanía política se injerta en el nivel más elemental del cuerpo social: entre sujeto y sujeto $-\mathrm{y}$ muchas veces se trata de los más humildes-, entre los miembros de una familia, en las relaciones de vecindad, de interés, de oficio, de rivalidad, de amor y de odio" (Foucault, 1996, p.131).

Con las determinaciones propuestas por la Reforma Radical y el Decreto orgánico de instrucción pública primaria, "lo social" se propone “como un campo construido, 'inventado' estratégicamente para producir, conducir, gestionar y determinar las llamadas 'necesidades o aspiraciones naturales' y las 'reivindicaciones de justicia' de la población, en términos de la redistribución de los beneficios económicos a través de 'servicios' -estatales o privados- de salud, educación, bienestar, seguridad social y servicios públicos" (Saldarriaga, 2003, p 208.). De esta manera, "las disciplinas del cuerpo y las regulaciones de la población constituyen los dos polos alrededor de los cuales se desarrolló la organización del poder sobre la vida" (Foucault, 1996, p. 168,169). Se da paso a la legitimidad del derecho positivo "(...) como una presencia visible y abierta por derecho a todos en la medida en que cada escuela construida y cada maestro formado eran la realización de la misión social del Estado" (Saldarriaga, 2003, p. 222).
A partir de la implementación de la nueva tecnologia política de reforma radical -reforma instruccionista, moral y social, plantea Saldarriaga (2003) -, se erige la figura del maestro como responsable de la formación moral (humanista), al tiempo que de la científica. Con esto se produce una agudización en el proceso de laicización y de desplazamiento del poder pastoral que hasta entonces había sido instaurado por la Iglesia católica. Con este giro, se propone al maestro como nuevo "director espiritual", pastor, tecnólogo moral y político del individuo, al tiempo que responsable de la enseñanza científica, asumida como práctica pedagógica modernizadora y civilizatoria. El Decreto orgánico de la instrucción pública primaria plantea que "los institutores públicos tienen plena autoridad sobre los niños en todo lo que se refiere a su educación, y deben vigilar incesantemente su conducta no sólo dentro de la escuela sino fuera de ella, excepto dentro de los limites de la casa paterna" (Decreto orgánico de la instrucción pública primaria citado por Saldarriaga, 2003).

En lo que respecta al periodo que va de 1900 a 1946 la instrucción y la educación pública responden, según Saldarriaga, a "los fines últimos de progreso, defensa de la raza y democratización de la cultura" (Saldarriaga, 1997, p. 400). A partir de allí, desde una propuesta práctica y social se forma un nuevo sujeto social.

Lo social, en Colombia, se propone como la constitución de un espacio de poder en donde se reacomodan las fuerzas y las fronteras del Estado, la Iglesia y la sociedad civil: "En este sentido lo social se nos hace visible como resultado de una descripción histórica que recons-truye los enfrentamientos entre conjuntos estratégicos -ética religiosa o ética civil, caridad cristiana filantropia, educación o instrucción, moral social, biológica o católica-, y describe los dispositivos o mecanismos concretos de funcionamiento de esos conjuntos, integrados por patronatos escolares, inspectores, campañas, textos, instituciones de examen, médicos escolares, congresos y sociedades, reglamentos, métodos pedagógicos, sistemas disciplinarios y demás" (Saldarriaga, 1997, p. 401).

\section{Conclusión}

El maestro en Colombia es sujetado por una serie de dispositivos de poder generadores de 
un saber que va a direccionar sus cuerpos desde una racionalidad gubernamental y tecnologias anatomo y biopolíticas que se encargan, de un lado, de disciplinar mediante mecanismos de control sus cuerpos y, de otro, de conducir al gremio como población asumida como especie.

En las dinámicas de subjetivación se presentan relaciones de poder en donde el sujeto maestro se convierte en objeto de disciplinamiento y control, a partir de las tecnologias de poder y las politicas de la vida. El sujeto maestro en su individualidad, es sujetado por las regulaciones políticas, estableciendo de este modo un vértice que une el poder con la vida.

La constitución del sujeto maestro no se debe a un solo factor, sino al choque y constante entrecruzamiento de múltiples prácticas y relaciones que se dinamizan a nivel macro y micropolítico.

El disciplinamiento del cuerpo del maestro, en la colonia se hacía, inicialmente, antes de que el Estado tuviera dominio sobre él, mediantela censura que efectúan no solo las autoridades politicas y religiosas virreinales, sino también por parte de las personas de bien que constituian los pilares del poder gubernamentlal en la época. Es el caso, primero, de la expulsión de los jesuitas y, segundo de los primeros maestros que ofrecian sus servicios de manera ambulante. La conducción de sus cuerpos se hace a través de dispositivos y prácticas jurídicas tales como las providencias y ordenanzas emanadas por el Gobierno de los Borbones. Ellos implementan una nueva gubernamentabilidad en aras de rescatar el poder, mediante la conducción de las poblaciones de manera directa sin que medie el poder de la Iglesia, pero sí contando con éste. Los modos de subjetivación del "sujeto maestro" se producen en los espacios del saber y del poder en órdenes del discurso, enunciaciones que se dan en prácticas históricas concretas que constituyen regimenes de verdad sociales, culturales, políticas y los ámbitos epistémicos de los saberes proferidos por diferentes objetos de saber y discursos emanados por diversos escuelas y/o autores traídos a nuestro país tales como los de Lancaster, Pestalozzi, Dewey $\mathrm{y}$ otros, que han constituido saber desde las ciencias, en interrelación con saberes y prácticas propias.
El análisis hecho sobre la base del anterior discurso, permite descubrir en última instancia, que dentro de la agencia del Estado, sus tendencias y lineas de poder, la administración de una gran seguridad molar (Deleuze, 2000) organizada a travês de tecnologias, dispositivos y estrategias, tiene como correlato una microgestión de pequeños miedos, una inseguridad molecular permanente, hasta el punto de que la fórmula de los ministerios del interior podría ser: una macropolítica de la sociedad para y por una micropolítica de la inseguridad que incide en la subjetivación del maestro en Colombia?.

Para finalizar, en el sentido de la reflexión sobre cómo se constituye la salud mental del sujeto maestro en Colombia, es preciso volver sobre la pregunta kantiana, rescatada por Foucault, para precisar su ontología crítica del presente y del sí mismo: ¿Qué somos en este tiempo? (Foucault, 2004). 


\section{Referencias}

1. Agamben, G. (2005). Conferencia ¿Qué es un dispositivo? Fuente: http:// libertaddepalabra.tripod.com/id11.html

2. Castro, S. (2005). La hybris del punto cero. Bogotá: Pontificia Universidad Javeriana.

3. Deleuze, G. (1987). Foucault. Barcelona: Paidós.

4. Deleuze, G. \& Guattari, F. (2000). Mil mesetas. Valencia: pretextos.

5. Foucault, M. (2006). Seguridad, territorio y población. Buenos Aires: Fondo de Cultura Económica.

6. Foucault, M. (2004). Tecnología política del individuo. Revista Veredas, número 9. Pags 213-226.

7. Foucault, M. (2002). Hermenéutica del sujeto. México D.F: Fondo de Cultura Económica.

8. Foucault, M. (2002). Nietzsche, la genealogía y la historia. Valencia: Pre-textos.

9. Foucault, M. (1996). Historia de la sexualidad. México D.F: Siglo XXI.

10. Foucault, M. (1999). La filosofía analítica de la política. En Estética, ética y hermenéutica. Barcelona: Paidós.

11. Foucault, M. (1996). La vida de los hombres infames. La Plata (Argentina): Caronte.

12. Foucault. M (1985). Saber y verdad. Madrid: La piqueta.

13. Herrán, S. (1998). Fundación del Colegio Máximo de la Compañía de Jesús y el colegio de San Bartolomé en el nuevo reino de granada. Revista Historia de la educación colombiana, pags 9-38 (1).

14. Cervantes Virtual. (S.d.). Pragmática sanción, para el estrañamiento de los reinos de la Compañía de Jesús. Consultado el 25 de junio de 2010, de http://www.cervantesvirtual.com/ bib_tematica/jesuitas/presentacion/ presentacion.shtml.

15. Martínez. B, Castro J. Noguera, C. (2002). Crónica del desarraigo. Bogotá: Magisterio.

16. Martínez, B. Silva R. (1984) Dos estudios sobre la educción en la colonia. Bogotá. cIUP - Universidad Pedagógica Nacional.

17. Saldarriaga, Ó. (2003). Del oficio de Maestro - Prácticas y teorías de la pedagogía moderna en Colombia. Bogotá: Magisterio.

18. Sáenz, J., Saldarriaga, O. \& Ospina, A. (1997). Mirar la infancia: pedagogía moral y modernidad en Colombia, 19031946. Bogotá: Colciencias.

19. Vásquez, L. (2007). Filosofía de la educación. Bogotá: Indoamerican Press Service. 
